\title{
Effects of Central Sensitivity Syndrome and Psychological Factors on the Clinical Features of Patients with Cervical Degenerative Disease: A Cross-Sectional Study
}

\author{
Yu Kondo ${ }^{1}$, Takahiro Miki ${ }^{1}$, Daisuke Higuchi ${ }^{2}$, Tsuneo Takebayashi ${ }^{3}$ \\ ${ }^{1}$ Department of Rehabilitation, Sapporo Maruyama Orthopedic Hospital, Sapporo, Japan \\ ${ }^{2}$ Department of Physical Therapy, Takasaki University of Health and Welfare, Takasaki, Japan \\ ${ }^{3}$ Department of Orthopaedic Surgery, Sapporo Maruyama Orthopedic Hospital, Sapporo, Japan
}

Study Design: Single-center cross-sectional study.

Purpose: The present study aimed to clarify the effects of central sensitivity syndrome (CSS) and psychological factors on the clinical features in patients with cervical degenerative disease (CDD).

Overview of Literature: The presence of CSS and psychological factors can influence the clinical features of patients with musculoskeletal disorders including CDD. However, the precise effects of CSS are unclear.

Methods: Patients admitted for surgical treatment of CDD were recruited. The following patient-reported outcome measures were recorded on the day before the surgery: the Numerical Rating Scale (NRS) for neck pain intensity, Neck Disability Index (NDI), EuroQol 5-Dimensions (EQ-5D) survey, Central Sensitization Inventory (CSI), Pain Catastrophizing Scale, Tampa Scale for Kinesiophobia, and the Hospital Anxiety and Depression Scale were used. We performed three multiple regression analyses to investigate the effects of CSS and psychological factors on the clinical features.

Results: Multiple regression analysis revealed that CSI had a significant effect on NRS ( $B, 0.50 ; 95 \%$ confidence interval [CI], 0.29 to 0.71 ), NDI ( $B, 0.64 ; 95 \% \mathrm{Cl}, 0.45$ to 0.82$)$, and $\mathrm{EQ}-5 \mathrm{D}(\mathrm{B},-0.55 ; 95 \% \mathrm{Cl},-0.75$ to -0.35$)$. Multiple regression analysis revealed that psychological factors did not exert a significant effect on the clinical features.

Conclusions: Our results demonstrated that CSI was able to identify the clinical features in CDD patients, suggesting that CSS does affect the clinical features of such patients.

Keywords: Spine; Neck; Cervical vertebrae; Neuropsychology

\section{Introduction}

Cervical diseases are a class of common musculosk- eletal disorders that are widely recognized as a major health problem [1]. Many people experience recurrent or chronic symptoms, resulting in increased health care

Received Apr 18, 2020; Revised Jul 1, 2020; Accepted Jul 4, 2020

Corresponding author: Takahiro Miki

Department of Rehabilitation, Sapporo Maruyama Orthopedic Hospital, N7-W27, Chuo-ku, Sapporo, Hokkaido 060-0007, Japan

Tel: +81-11-612-1133, Fax: +81-11-612-8151, E-mail: tkhr.mk@gmail.com 
costs and social loss [2]. Among the cervical diseases, cervical degenerative disease (CDD) affects the spinal cord and nerve roots, sometimes causing neuropathic pain. Although the efficacy of conservative therapy has been reported for mild cases [3], surgical treatment is often required for severe or progressive cases of CDD. However, even if surgery is performed properly and the pressure on the nerve is released, postoperative residual pain is often experienced [4]. The pathology of CDD is complex, and the underlying mechanisms that contribute to pain management and recovery are incompletely understood.

Recently, central sensitization (CS) has been suggested as a condition of chronic pain. The International Association for the Study of Pain defines CS as increased responsiveness of nociceptive neurons in the central nervous system to their normal or subthreshold afferent input [5]. Multiple factors, including psychological factors, may cause dysregulation of bottom-up or top-down modulation and cause CS [6]. Well-known psychological factors involved in CS include pain catastrophizing, depression, fear, anxiety, stress, and inadequate knowledge of the respective disease. In support of this, previous studies have reported that negative emotion impairs the descending inhibitory pathways [7].

Many conditions of chronic pain, such as low back pain [8] as well as neck and shoulder pain [9] are characterized by hypersensitivity induced by CS. Yunus [10] proposed the idea of an overlapping and similar group of syndromes bound by the common mechanisms of CS, called central sensitivity syndromes (CSS). Recent investigations have shown that CSS patients may also have the clinical features in lumbar disease [8]. Moreover, CSS patients are reported to have significantly lower values for catastrophic thinking, fear of exercise, and sleep effects than controls for patients with neck and shoulder pain [9].

A previous study has reported the effects of psychological factors in the development of CDD [11-13]. However, to our knowledge, no studies have focused on the influence of CSS in terms of the development of CDD, and the precise effects of CSS are unclear. CSS may affect the clinical symptoms of CDD. The present study aimed to investigate the effects of CSS and the psychological factors on the clinical features of CDD patients.

\section{Materials and Methods}

\section{Design}

This single-center, cross-sectional study and all its protocols were approved by the Ethics Committee of Sapporo Maruyama Orthopedic Hospital (approval no., \#000028). Written informed consent was obtained from each participant before initiation data collection.

\section{Participants}

All CDD patients who were referred to the Sapporo Maruyama Orthopedic Hospital (Sapporo, Japan) were invited to participate in this study. The inclusion criteria were as follows: (1) undergoing cervical spine surgery following medical diagnosis of cervical spondylotic myelopathy, cervical spondylotic radiculopathy, cervical disk herniation, or ossification of the posterior longitudinal ligamentum between May 2018 and March 2020; (2) age 20-90 years; and (3) evaluable on the day before the surgery. Exclusion criteria were as follows: (1) serious pathologies, such as cerebrovascular or cardiovascular disease; (2) history of mental disorder; (3) history of cervical spine surgery; and (4) difficulty in answering the questionnaires.

\section{Procedures}

The following patient-reported outcome measures (PROMs) were evaluated on the day before the surgery for all the participants: (1) Numerical Rating Scale (NRS) for neck pain intensity, (2) Neck Disability Index (NDI), (3) EuroQol 5-Dimensions (EQ-5D) survey of healthrelated quality of life (HRQOL), (4) Central Sensitization Inventory (CSI) for CSS, (5) Pain Catastrophizing Scale (PCS), (6) Tampa Scale for Kinesiophobia (TSK), and (7) Hospital Anxiety and Depression Scale (HADS). Data regarding age, sex, height, weight, medical diagnosis, and symptom duration were collected from the patients' medical records.

CSI was developed to identify patients who may have CSS [14]. Subsequently, the ability to identify CSS was verified [15]. All other questionnaires are highly reliable, with the reliability and validity of the Japanese version being confirmed. This questionnaire was selected because it is used internationally and can be used for comparing the 
present findings with previous reports. The details are as follows.

\section{Measures}

1) Numerical Rating Scale for neck pain intensity

Pain intensity was measured using the 11-point NRS to determine average neck pain intensity on the day of the evaluation. A score of 0 indicates no pain, and a score of 10 indicates the worst imaginable pain.

\section{2) Neck Disability Index}

Disability was measured using the Japanese version of the NDI [16]. The NDI includes 10 items, scored from 0 to 5 . The total score is converted to a percentage, with a higher total score indicating greater disability. Previous studies have demonstrated the high internal consistency (Cronbach's $\alpha$ of all items $=0.89$ ) of the Japanese version of the NDI [16].

\section{3) EuroQol 5-Dimensions}

We measured the HRQOL using the Japanese version of the EQ-5D [17]. The EQ-5D consists of 5 dimensions relating to the state of health: mobility, self-care, usual activities, pain/discomfort, and anxiety/depression. A higher score indicates a better quality of life, with a maximum score of 1 [18].

\section{4) Central Sensitization Inventory}

The Japanese version of the CSI was used to assess CSS [19]. The CSI includes 25 items, scored from 0 to 4 (Table 1) [14]. A higher total score indicates more severe CSS, with a total score of 40 being the cutoff [14]. Previous studies have demonstrated this system to exhibit good criterion validity, construct validity, and test-retest reliability (intra-class correlation coefficient $=0.85$ ) [19].

\section{5) Pain Catastrophizing Scale}

Pain catastrophizing was measured using the Japanese version of the PCS that includes 13 items, with scores ranging from 0 to 4 [20]. A higher total score indicates a greater degree of pain catastrophizing, and a total score of 30 is the cutoff point for clinically relevant level of catastrophizing [21]. Previous studies have demonstrated the construct validity and high internal consistency (Cronbach's $\alpha$ of all items $=0.89$ ) of the Japanese version of the PCS [20].
Table 1. Central Sensitization Inventory [14]

\begin{tabular}{|c|c|}
\hline Item no. & Central Sensitization Inventory \\
\hline 1 & I feel tired and unrefreshed when I wake from sleeping. \\
\hline 2 & My muscles feel stiff and achy. \\
\hline 3 & I have anxiety attacks. \\
\hline 4 & I grind or clench my teeth. \\
\hline 5 & I have problems with diarrhea and/or constipation. \\
\hline 6 & I need help in performing my daily activities. \\
\hline 7 & I am sensitive to bright lights. \\
\hline 8 & I get tired very easily when I am physically active. \\
\hline 9 & I feel pain all over my body. \\
\hline 10 & I have headaches. \\
\hline 11 & I feel discomfort in my bladder and/or burning when I urinate. \\
\hline 12 & I do not sleep well. \\
\hline 13 & I have difficulty concentrating. \\
\hline 14 & I have skin problems such as dryness, itchiness, or rashes. \\
\hline 15 & Stress makes my physical symptoms get worse. \\
\hline 16 & I feel sad or depressed. \\
\hline 17 & I have low energy. \\
\hline 18 & I have muscle tension in my neck and shoulders. \\
\hline 19 & I have pain in my jaw. \\
\hline 20 & $\begin{array}{l}\text { Certain smells, such as perfumes, make me feel dizzy and nause- } \\
\text { ated. }\end{array}$ \\
\hline 21 & I have to urinate frequently. \\
\hline 22 & $\begin{array}{l}\text { My legs feel uncomfortable and restless when I am trying to go to } \\
\text { sleep at night. }\end{array}$ \\
\hline 23 & I have difficulty remembering things. \\
\hline 24 & I suffered trauma as a child. \\
\hline 25 & I have pain in my pelvic area. \\
\hline
\end{tabular}

6) Tampa Scale for Kinesiophobia

Kinesiophobia was measured using the Japanese version of the TSK [22]. The TSK includes 17 items, scored from 1 to 4 . A higher total score indicates greater kinesiophobia, and a total score of 37 is the cutoff point that relates to strong kinesiophobia [22]. Previous studies have demonstrated the concurrent validity and high internal consistency (Cronbach's $\alpha$ of all items $=0.85$ ) of the Japanese version of the TSK [22].

7) Hospital Anxiety and Depression Scale

Anxiety or depression was measured using the Japanese version of the HADS [23]. The HADS includes 14 items, with scores ranging from 0 to 3 . Odd number items are for depression, and the even number items are for anxiety. A 
higher total score indicates greater anxiety or depression. In each subscale of anxiety or depression, a total score of 8 is the cutoff point for the identification of suspicious cases for depression and anxiety [23]. Previous studies have demonstrated the construct validity of the Japanese version of the HADS [23].

\section{Statistical analyses}

Missing values were imputed using the multiple imputation with the chained equation method. Descriptive analyses were used to evaluate participant characteristics. Continuous data are expressed as the mean (standard deviation) values and categorical data as counts (percentages). Correlations between CSI and psychological factors (pain catastrophizing, kinesiophobia, anxiety, depression) and clinical features were examined using Spearman's rank correlation coefficient. Three multiple regression analyses were then performed to assess the value of CSI and PROMs for psychological factors to identify NRS, NDI, and EQ-5D. The CSI and psychological factors were entered as independent variables, along with potentially important variables, such as age and symptom duration, regardless of statistical significance. In the present study, $r$-values of $\leq 0.40,0.40-0.75$, or $>0.75$ were considered to indicate weak or no correlation, moderate correlation, and strong correlation, respectively [24]. Multiple imputation was performed using R ver. 3.6 (The R Foundation for Statistical Computing, Vienna, Austria; https:// www.r-project.org/), and other statistical analyses were performed using HAD ver. 16.1 (Hiroshi Shimizu, Nishinomiya, Japan). A significance level of 0.05 was selected; however, we primarily focused on point estimates and 95\% confidence intervals (95\% CI) to evaluate the significance of the results.

\section{Results}

\section{Participant characteristics}

Seventy-two patients with CDD were included based on the aforementioned inclusion criteria. Of the study population, $6.9 \%(5 / 72)$ had incomplete data, representing $2 \%$ of the 86 questions $(121 / 6,002)$. These missing values were complemented with the multiple imputation method. In total, the data of 72 patients were analyzed. Of these, 42 patients were treated for cervical spondylotic myelopathy,
Table 2. Characteristics of the study population $(n=72)$

\begin{tabular}{|c|c|}
\hline Characteristic & Value \\
\hline Female gender & $20(28.0)$ \\
\hline Age (yr) & $64.2 \pm 11.0$ \\
\hline Body mass index $\left(\mathrm{kg} / \mathrm{m}^{2}\right)$ & $24.5 \pm 3.9$ \\
\hline Duration of symptoms (mo) & $27.8 \pm 46.1$ \\
\hline Numerical Rating Scale for neck pain intensity (0-10) & $3.0 \pm 2.7$ \\
\hline Neck Disability Index & $26.9 \pm 17.4$ \\
\hline Euro0ol 5-Dimensions (0.00-1.00) & $0.65 \pm 0.11$ \\
\hline Central Sensitization Inventory (0-100) & $26.2 \pm 12.9$ \\
\hline Cutoff score for Central Sensitization Inventory of $\geq 40$ & $15(20.1)$ \\
\hline Pain Catastrophizing Scale (0-52) & $28.0 \pm 11.1$ \\
\hline Cutoff score for Pain Catastrophizing Scale of $\geq 30$ & $32(44.4)$ \\
\hline Tampa Scale for Kinesiophobia (17-68) & $41.5 \pm 6.2$ \\
\hline Cutoff score for Tampa Scale for Kinesiophobia of $\geq 37$ & $61(85.0)$ \\
\hline Hospital Anxiety and Depression Scale-anxiety subscale (0-21) & $6.0 \pm 3.4$ \\
\hline $\begin{array}{l}\text { Cutoff score for Hospital Anxiety and Depression Scale-anxiety } \\
\text { subscale of } \geq 8\end{array}$ & $22(30.6)$ \\
\hline $\begin{array}{l}\text { Hospital Anxiety and Depression Scale-depression subscale } \\
(0-21)\end{array}$ & $6.2 \pm 3.9$ \\
\hline $\begin{array}{l}\text { Cutoff score for Hospital Anxiety and Depression Scale-depres- } \\
\text { sion subscale of } \geq 8\end{array}$ & $25(35.0)$ \\
\hline
\end{tabular}

18 for cervical spondylotic radiculopathy, four for cervical disc herniation, and seven for ossification of the posterior longitudinal ligamentum. The characteristics of the study population are summarized in Table 2 .

\section{Correlation analysis}

Table 3 presents the correlation between CSI and PROMs for psychological factors and clinical features. We found that CSI was significantly and moderately correlated with NRS $(r=0.50, p<0.01)$, NDI $(r=0.64, p<0.01)$, and EQ-5D $(r=-0.55, p<0.01)$. Both TSK and HADS were significantly correlated with some clinical features.

\section{Multiple regression analyses}

Tables 4-6 present the results of multiple regression models with NRS, NDI, and EQ-5D as the dependent variables, respectively. The constructed model for the identification of NRS, NDI, and EQ-5D significantly fits the overall data $\left(R^{2}=0.25\right.$, adjusted $R^{2}=0.24, p<0.01 ; R^{2}=0.40$, adjusted $R^{2}=0.40, p<0.01$; and $R^{2}=0.30$, adjusted $R^{2}=0.29$, 
Table 3. Correlations between clinical features and evaluated variables

\begin{tabular}{lccccccc} 
& 1 & 2 & 3 & 4 & 5 & 6 & 7 \\
1. NRS & - & - & - & - & - & - & - \\
2. NDI & $0.69^{* *}$ & - & - & - & - & - & - \\
3. EQ-5D & $-0.33^{*}$ & $-0.57^{* *}$ & - & - & - & - & - \\
4. CSI & $0.50^{* *}$ & $0.64^{* *}$ & $-0.55^{* *}$ & - & - & - & - \\
5. PCS & 0.30 & 0.18 & -0.20 & $0.29^{*}$ & - & - \\
6. TSK & 0.14 & $0.26^{*}$ & $-0.44^{* *}$ & $0.47^{* *}$ & $0.53^{* *}$ & - & - \\
\hline 7. HADS-A & 0.19 & $0.34^{* *}$ & $-0.38^{*}$ & $0.50^{*}$ & $0.51^{* *}$ & $0.38^{*}$ & - \\
8. HADS-D & 0.13 & $0.27^{*}$ & $-0.23^{* *}$ & $0.47^{*}$ & $0.40^{* *}$ & 0.23 & - \\
\hline
\end{tabular}

NRS, Numerical Rating Scale for neck pain intensity; NDI, Neck Disability Index; EQ-5D, EuroQol 5-Dimensions; CSI, Central Sensitization Inventory; PCS, Pain Catastrophizing Scale; TSK, Tampa Scale for Kinesiophobia; HADS-A, Hospital Anxiety and Depression Scale for anxiety; HADS-D, Hospital Anxiety and Depression Scale for depression.

" $p<0.05$ and ${ }^{* *} p<0.01$ (by Spearman's rank correlation coefficient).

Table 4. Results of multiple regression analysis with pain intensity as the dependent variable

\begin{tabular}{ccccccc} 
& $B$ & SE & $\boldsymbol{\beta}$ & $T$-value & $p$-value & $95 \% \mathrm{Cl}$ \\
$\mathrm{CSI}$ & 0.10 & 0.02 & 0.50 & 4.83 & $<0.01$ & $0.29-0.71$ \\
\hline
\end{tabular}

$R^{2}=0.25$; adjusted $R^{2}=0.24$.

$B$, partial regression coefficient; $\mathrm{SE}$, standard error; $\beta$, standardized partial regression coefficient; $95 \% \mathrm{Cl}, 95 \%$ confidence interval of the estimated effect; CSI, Central Sensitization Inventory.

Table 5. Results of multiple regression analysis with disability as the dependent variable

$\begin{array}{ccccccc} & B & \mathrm{SE} & \boldsymbol{\beta} & T \text {-value } & p \text {-value } & 95 \% \mathrm{Cl} \\ \mathrm{CSI} & 0.86 & 0.12 & 0.64 & 6.90 & <0.01 & 0.45-0.82\end{array}$

$R^{2}=0.40$; adjusted $R^{2}=0.40$.

$B$, partial regression coefficient; $\mathrm{SE}$, standard error; $\beta$, standardized partial regression coefficient; $95 \% \mathrm{Cl}, 95 \%$ confidence interval of the estimated effect; CSI, Central Sensitization Inventory.

Table 6. Results of multiple regression analysis with health-related quality of life as the dependent variable

\begin{tabular}{ccccccc} 
& $B$ & SE & $\boldsymbol{\beta}$ & $T$-value & $p$-value & $95 \% \mathrm{Cl}$ \\
CSI & -0.01 & 0.001 & -0.55 & -5.52 & $<0.01$ & -0.75 to -0.35 \\
\hline
\end{tabular}

$R^{2}=0.30$; adjusted $R^{2}=0.29$.

$B$, partial regression coefficient; $S E$, standard error; $\beta$, standardized partial regression coefficient; $95 \% \mathrm{Cl}, 95 \%$ confidence interval of the estimated effect; CSI, Central Sensitization Inventory.

$p<0.01)$, respectively. CSI has a significant effect on NRS $(\beta, 0.50 ; 95 \% \mathrm{CI}, 0.29$ to 0.71$)$, NDI $(\beta, 0.64 ; 95 \% \mathrm{CI}, 0.45$ to 0.82 ), and EQ-5D ( $\beta,-0.55 ; 95 \% \mathrm{CI},-0.75$ to -0.35$)$. The PROMs for psychological factors did not significantly affect the clinical features. There were no associations with multicollinearity (variance inflation factor $<3.0$ ), indicating that the results were reliable.

\section{Discussion}

This study revealed that CSI is a significant identifying factor for the clinical features, suggesting that CSS has a significant effect on the clinical features of CDD patients.

Previous studies have investigated the effects of CSS in lumbar disease; Bennett et al. [25] found that preoperative CSI is associated with reduced HRQOL and increased length of hospital stay after lumbar spinal fusion. In particular, for every 10-point increase in the CSI score, the length of hospital stay increased by $6.4 \%$. Neblett et al. [8] reported that the CSI score at the time of admission for chronic spinal pain is associated with pain intensity and that interdisciplinary biopsychosocial treatment can significantly improve the CSI score.

Multiple regression analysis in the present study revealed that the CSI score was significant for identifying the pain intensity, disability, and HRQOL. Therefore, after controlling for other confounding factors, we suggest that more severe CSS is associated with more severe clinical features in CDD patients. Our conclusions are consistent with previous studies in terms of the effects of CSS on the clinical features of patients with lumbar disease $[8,25]$.

The present analysis of the correlations between psychological factors and clinical features revealed that TSK and HADS were significantly correlated with some clinical features. However, these factors did not affect the clinical 
features when CSI and psychological factors were used simultaneously as independent variables in multiple regression analysis.

Previous studies have analyzed the effects of psychological factors on the clinical features of CDD patients. In their evaluation of depression using the Patient Health Questionnaire-9 following posterior cervical fusion, Alvin et al. [11] demonstrated, through multiple linear and logistic regression analyses, that more severe preoperative depression is associated with a worse EQ-5D score at 1 year postoperatively. Archer et al. [12] reported that the preoperative symptoms of depression predict increased pain interference and disability and decreased HRQOL at 6 months after the surgery for lumbar or cervical degenerative conditions. In contrast, Doi et al. [13] found that cervical spinal fusion is associated with significant improvement of HRQOL and sufficient levels of satisfaction after the surgery, regardless of the presence of preoperative depression or anxiety in CDD patients.

Thus far, there is no consensus of the influence of psychological factors on CDD development. The results of previous studies about the effects of psychological factors in CDD patients do not account for CSS. Several studies have reported that pain intensity could be associated with the CSI score as well as psychological factors $[8,26]$. Shigetoh et al. [27] found that CS mediates the relationship between pain intensity and psychological factors including depression. Van Wilgen et al. [26] demonstrated the convergent validity of CSI score by evaluating the clinical features of CS and psychological factors. In addition to primary symptoms associated with CSS, the CSI also briefly assesses psychological factors, including anxiety, depression, and panic disorder. To our knowledge, no previous studies have simultaneously investigated the effects of CSS and psychosocial factors in CDD patients. The present study suggests that CSI may reflect clinical features to a better degree than PROMs for psychological factors.

For the clinical implication, treatment of CSS should focus on multidisciplinary biopsychosocial treatment and medications, such as dual reuptake inhibitors $[8,14]$. A high CSI score should not preclude surgical treatment; however, clinicians should select their patients carefully and follow different treatment policies as per need. Our findings highlight the utility of the CSI score for identifying the severity of clinical features among such patients. The addition of CSI that comprehensively assesses psychological factors has the potential to help diagnose patients with CDD and develop an appropriate treatment plan.

The present study has certain limitations that need to be acknowledged. The first is that CSS was evaluated using the CSI score; the diagnosis of CSS could not be established using more objective evaluations. In fact, the PROM score might have been affected by age, cognition, and cultural aspects, such as the fact that the population was Japanese. Quantitative Sensory Testing (QST) has been used to assess CSS in order to quantify somatosensory function [28]. Although a correlation between clinical symptoms measured using QST and CSS has been reported [29], QST is often thought of as a direct indicator of CSS. Therefore, it may be more reliable to measure QST in this study. The second limitation is the relatively small sample size. Total 72 participants were selected; however, the number of participants was insufficient. Further large studies are needed in order to confirm our findings. Finally, this was a cross-sectional study; therefore, a causal relationship between CSI and clinical features cannot be confirmed. Prospective studies are required to validate the CSI score as a screening tool for cervical spine surgery. For example, it has been reported that preoperative CSI scores in people with lumbar disease had prolonged discharge and lower HRQOL [25]. Therefore, it is necessary to examine how the preoperative CSI scores affect postoperative outcomes in cervical disease.

\section{Conclusions}

This study investigated the effects of CSS and psychological factors on the clinical features of CDD patients with CDD. We found that CSI can help identify clinical features, suggesting that CSS affects pain intensity, disability and HRQOL of CDD patients. Our findings may facilitate clinical decision making because they highlight the importance of evaluating CSI in the clinic for identifying the severity of clinical features of CDD patients and plan appropriate management strategies.

\section{Conflict of Interest}

No potential conflict of interest relevant to this article was reported.

\section{Acknowledgments}

The authors would like to thank Crimson Interactive Ja- 
pan Co., Ltd. (https://www.enago.jp) for the English language editing.

\section{ORCID}

Yu Kondo: https://orcid.org/0000-0003-3567-5676; Takahiro Miki: https://orcid.org/0000-0002-0648-2675; Daisuke Higuchi: https://orcid.org/0000-0001-7197-6068

\section{Author Contributions}

Conceptualization: YK, TM; data curation: YK, TM; formal analysis: DH; investigation: YK, TM; methodology: YK, TM, DH; project administration: TT; supervision: TM, TT; visualization: YK, TM; and writing-original draft: YK, TM.

\section{References}

1. Gross A, Kay TM, Paquin JP, et al. Exercises for mechanical neck disorders. Cochrane Database Syst Rev 2015;1:CD004250.

2. Hill J, Lewis M, Papageorgiou AC, Dziedzic K, Croft P. Predicting persistent neck pain: a 1-year followup of a population cohort. Spine (Phila Pa 1976) 2004;29:1648-54.

3. Kadanka Z, Bednarík J, Vohanka S, et al. Conservative treatment versus surgery in spondylotic cervical myelopathy: a prospective randomised study. Eur Spine J 2000;9:538-44.

4. Higuchi D. Prognostic value of preoperative coping strategies for pain in patients with residual neuropathic pain after laminoplasty for compressive cervical myelopathy. Asian Spine J 2015;9:675-82.

5. Loeser JD, Treede RD. The Kyoto protocol of IASP Basic Pain Terminology. Pain 2008;137:473-7.

6. Van Wijk G, Veldhuijzen DS. Perspective on diffuse noxious inhibitory controls as a model of endogenous pain modulation in clinical pain syndromes. J Pain 2010;11:408-19.

7. De Souza JB, Potvin S, Goffaux P, Charest J, Marchand $\mathrm{S}$. The deficit of pain inhibition in fibromyalgia is more pronounced in patients with comorbid depressive symptoms. Clin J Pain 2009;25:123-7.

8. Neblett R, Hartzell MM, Williams M, Bevers KR, Mayer TG, Gatchel RJ. Use of the Central Sensi- tization Inventory (CSI) as a treatment outcome measure for patients with chronic spinal pain disorder in a functional restoration program. Spine J 2017;17:1819-29.

9. Sjors A, Larsson B, Persson AL, Gerdle B. An increased response to experimental muscle pain is related to psychological status in women with chronic non-traumatic neck-shoulder pain. BMC Musculoskelet Disord 2011;12:230.

10. Yunus MB. Fibromyalgia and overlapping disorders: the unifying concept of central sensitivity syndromes. Semin Arthritis Rheum 2007;36:339-56.

11. Alvin MD, Miller JA, Sundar S, et al. The impact of preoperative depression on quality of life outcomes after posterior cervical fusion. Spine J 2015;15:79-85.

12. Archer KR, Seebach CL, Mathis SL, Riley LH 3rd, Wegener ST. Early postoperative fear of movement predicts pain, disability, and physical health six months after spinal surgery for degenerative conditions. Spine J 2014;14:759-67.

13. Doi T, Nakamoto H, Nakajima K, et al. Effect of depression and anxiety on health-related quality of life outcomes and patient satisfaction after surgery for cervical compressive myelopathy. J Neurosurg Spine 2019:1-8.

14. Mayer TG, Neblett R, Cohen H, et al. The development and psychometric validation of the central sensitization inventory. Pain Pract 2012;12:276-85.

15. Neblett R, Hartzell MM, Cohen H, et al. Ability of the central sensitization inventory to identify central sensitivity syndromes in an outpatient chronic pain sample. Clin J Pain 2015;31:323-32.

16. Takeshita K, Hosono N, Kawaguchi Y, et al. Validity, reliability and responsiveness of the Japanese version of the Neck Disability Index. J Orthop Sci 2013;18:14-21.

17. Shiroiwa T, Ikeda S, Noto S, et al. Comparison of value set based on DCE and/or TTO data: scoring for EQ-5D-5L health states in Japan. Value Health 2016;19:648-54.

18. Van Hout B, Janssen MF, Feng YS, et al. Interim scoring for the EQ-5D-5L: mapping the EQ-5D-5L to EQ-5D-3L value sets. Value Health 2012;15:708-15.

19. Tanaka K, Nishigami T, Mibu A, et al. Validation of the Japanese version of the Central Sensitization Inventory in patients with musculoskeletal disorders. PLoS One 2017;12:e0188719. 
20. Matsuoka H, Sakano Y. Assessment of cognitive aspect of pain: development, reliability, and validation of Japanese version of pain catastrophizing scale. Jpn J Psychosom Med 2007;47:95-102.

21. Sullivan MJ. The pain catastrophizing scale: user manual. Montreal: McGill University; 2009.

22. Kikuchi N, Matsudaira K, Sawada T, Oka H. Psychometric properties of the Japanese version of the Tampa Scale for Kinesiophobia (TSK-J) in patients with whiplash neck injury pain and/or low back pain. J Orthop Sci 2015;20:985-92.

23. Matsudaira T, Igarashi H, Kikuchi H, et al. Factor structure of the Hospital Anxiety and Depression Scale in Japanese psychiatric outpatient and student populations. Health Qual Life Outcomes 2009;7:42.

24. Andresen EM. Criteria for assessing the tools of disability outcomes research. Arch Phys Med Rehabil 2000;81(12 Suppl 2):S15-20.

25. Bennett EE, Walsh KM, Thompson NR, Krishnaney AA. Central sensitization inventory as a predictor of worse quality of life measures and increased length of stay following spinal fusion. World Neurosurg 2017;104:594-600.
26. Van Wilgen CP, Vuijk PJ, Kregel J, et al. Psychological distress and widespread pain contribute to the variance of the central sensitization inventory: a crosssectional study in patients with chronic pain. Pain Pract 2018;18:239-46.

27. Shigetoh H, Tanaka Y, Koga M, Osumi M, Morioka S. The mediating effect of central sensitization on the relation between pain intensity and psychological factors: a cross-sectional study with mediation analysis. Pain Res Manag 2019;2019:3916135.

28. Correa JB, Costa LO, de Oliveira NT, Sluka KA, Liebano RE. Central sensitization and changes in conditioned pain modulation in people with chronic nonspecific low back pain: a case-control study. Exp Brain Res 2015;233:2391-9.

29. Van Wilgen CP, Konopka KH, Keizer D, Zwerver J, Dekker R. Do patients with chronic patellar tendinopathy have an altered somatosensory profile?: a Quantitative Sensory Testing (QST) study. Scand J Med Sci Sports 2013;23:149-55. 